\title{
Methodological Constrains in Aids Study
}

\author{
Sengottuvelu. $\mathrm{S}^{1 *}$, Priscilla Mary James ${ }^{2}$, Saravanan $\mathrm{R}^{3}$, \\ Padmapriya. $\mathrm{B}^{4}$ \\ ${ }^{1}$ Professor Department of Pharmacology, Nandha College of Pharmacy, Erode, \\ Tamilnadu, Email:sehejan@gmail.com \\ ${ }^{2}$ Assistant Professor, Department of Pharmacy Practice, Nandha College of Pharmacy, \\ Erode, Tamilnadu, Email:priscillamary1995@gmail.com \\ ${ }^{3}$ Assistant Professor, Department of Pharmacology, Nandha College of Pharmacy, \\ Erode, Tamilnadu, Email: saravanan@nandhapharmacy.org \\ ${ }^{4}$ Assistant Professor, Department of Community Health Nursing, Nandha College of \\ Nursing, Erode, Tamilnadu, Email:padmapriyapushpa@gmail.com \\ ${ }^{*}$ Corresponding Author
}

\begin{abstract}
The impact of HIV/AIDS on people's livelihoods has been much discussed from an outsider point of view rather than an insider point of view and culture-specific perspective. The insider explanation that is shared by a particular group of people, but which can differ between men and women, the young and the old. In contrast, the insider point of view is the scientific construction of emic elements that are verified by researchers through evidence. In this research paper there different elements are discussed to understand the emic and etic in the AIDS impact. Various methodological constrains are discussed to study and give clear understanding of the health problem in AIDS infection from various point of view.
\end{abstract}

Keywords: emic and etic, AIDS infection, scientific construction, methodological constrains.

\section{Introduction}

The work structure is a valuable instrument for sway research as it assists with investigating individuals' reactions with the impacts of the plague based on their resources and the resulting occupation results. It helps in getting sorted out data and presents specialists and improvement professionals from various orders with a typical reference. The ideas of emic and etic have assumed a significant job in the field of clinical human sciences and brought about the qualification among infection and disease. Ailment is emic as it depends on individuals own insight and sickness is etic as it depends on a logical definition. An emic portrayal of AIDS identifies with the experience and affiliations individuals have with AIDS. An emic model of AIDS is certainly not a static one and changes as mixed media missions and wellbeing laborers open individuals to the etic understandings of AIDS. Albeit these days numerous individuals know about the etic model of how disease functions and how AIDS creates, it doesn't give individuals answers on why they have been contaminated or are wiped out with AIDS. While applying an emic-etic qualification to the effect of AIDS on individuals' abilities, the accentuation of the individuals included may vary from the logical perspective. The etic model of AIDS sway depends on various subjective and quantitative examinations.
The intricacy of the structure likewise prompts issues of estimation and choice of significant pointers. The work structure makes the propensity to oversee intricacy by dividing it into unmistakable classifications that are isolated dissected for impacts .On justification of AIDS-sway research, the utilization of a business structure chances creating an extensive rundown of impacts and changes in the resource base, occupation systems and vocation results that could possibly be credited to AIDS. Therefore, the end-client may suffocate in the discoveries and may think that it's hard to locate the correct passage point for proper intercessions. To evade this, focusing on a restricted subset of potential effects. A scope of markers one can use to gauge and screen the effect of HIV/AIDS on the two family units and networks. The markers he recommended for estimating AIDS sway focus dominatingly on job resources and not straightforwardly on work results, for example, food security, since vocation resources are viewed as proximate determinants of family food security. Since alleviation techniques for AIDS sway on food security will most likely zero in on fortifying the resource base of families, it is likewise essential to distinguish first the impacts of AIDS on the diverse natural ability capitals. 


\section{Etic viewpoints issues in AIDS}

Moral issues occur at all periods of assessment, including the assurance of the investigation domain and assessment subjects, picking the wellspring of financing, coordinating the hands on work, the interpretation, examination and dispersal of results and the expulsion of data. Given the affectability of the topic, research on the impact of HIV/AIDS raises a couple of good issues. For example, an assessment on AIDS influence will without a doubt present individuals fragile requests concerning the infection just as death of family members, which may cause people pressure and fear and may have wide effects that are not predicted by the subject matter experts. Since AIDS will when all is said in done pack in nuclear families, it is routinely difficult to find a colossal enough illustration of impacted families, especially in locales with low prevalence rates. This may incite different undertakings to separate affected nuclear families, for instance, working through a close by prosperity network or expert community or the usage of clinical administrations systems based testing. While social order and other close by prosperity workers have a nice data on the different nuclear families, these families most likely won't have given their consent to the prosperity spot to confer their status to others or they presumably won't have an idea about their status themselves. Moral issues furthermore expect a work when examiners are endeavoring to improve enduring quality in distinctive adults who experience the evil impacts of HIV/AIDS. To do accordingly, experts have used a modified verbal analyzing method where information is assembled from close relatives or friends about the terminated signs going before demise Existing plots for research

The moral rule regard for people has to do with regarding and securing the privileges of people engaged with the exploration. The standard considers people to be independent people that reserve the option to choose whether or not to take an interest in a given examination. It says that before an investigation is started, individual educated assent from members should be acquired. The International Ethical Guidelines drafted by the Council for International Organizations of Medical Sciences, a member of the World Health Organization that considers moral issues in examination including human subjects, characterize educated assent as an authorization allowed by an able individual in the wake of getting and understanding the fundamental data without coercion to any type of pressing factor, impact, power or terrorizing. To settle on an allaround educated choice, members ought to get data on the motivation behind the examination, the cycle of the investigation, their job in the examination, regardless of whether the investigation offers advantages to members or to other people and the probability and nature of mental or social dangers in a reasonable language.
Helpfulness implies that scientists are dependable to shield their members from hurt and to boost potential advantages. While the motivation behind information assortment is to profit society, exploration ought not endanger the mental prosperity of people or put people in danger of social disgrace and segregation, and accordingly scientists should envision and forestall any likely contrary results. Under this guideline, exploration ought to have a substantial and unmistakably characterized reason. On interpretation of HIV/AIDS-sway examines, the intention is to comprehend the impacts of AIDS, the determinants of family's powerlessness to react, and to characterize regions to diminish their weakness to AIDS sway. Examination with regards to HIV/AIDS will in general include questions that are delicate, individual, and maybe hard to answer truly, notwithstanding, no inquiries ought to be forced that are obtrusive, embarrassing or accusatory.

\section{Limits to methodological constrains}

With concerns to AIDS-sway research, no unique codes of morals have yet been drafted. a direction archive on moral contemplations in HIV preventive immunization research, however these are biomedical situated. Most moral standards and rules, as depicted above, were initially produced for biomedical exploration, however they have been applied to social and conduct research also, including AIDS-sway examines. While these moral standards may sound straightforward, their real application in AIDS-sway research is testing and may cause clashes. Since HIV/AIDS is regularly connected with blood, passing, (business) sex, homosexuality and sexual savagery, it is hard to straightforwardly examine AIDS in numerous social orders.

Identified with the rule of value, is the issue of AIDS intermediary pointers and verbal examination techniques that are utilized to recognize AIDSinfluenced family units. Since the HIV status of an individual is private and all people reserve the option to control individual data or in light of the fact that individuals don't have the vaguest idea about their status, authority examines utilize elective estimates like intermediary markers and verbal dissection techniques to show whether somebody is associated with being HIV positive or having passed on from AIDS. By and by, this implies analysts are joining names to sicknesses or reasons for death. A moral worry that presently emerges is that when AIDS is ascribed to somebody's name on the grounds that the individual satisfies the conditions set by a specific intermediary marker, would we say we are to tell this individual? Not illuminating somebody on the probability regarding being tainted makes hurt people as they can't profit by treatment, just as to other people who danger turning out to be guiltless casualties. While numerous examinations exist on the effect of HIV/AIDS on individuals' occupations, small announcing has been done on the moral parts of the 


\section{exploration.}

It is indistinct from the investigations whether research proposition and polls were examined by a (neighborhood) research morals advisory group, how privacy was guaranteed and whenever educated assent was acquired. Report that in just one out of the 40 investigations they inspected it was unequivocally expressed that educated assent was (verbally) gotten from the HIV-positive individuals taking an interest in the examination. Exceptional difficulties to applying moral standards, yet this doesn't legitimize any suspension of the privileges of examination members to full data, educated assent, or insurance of classification. Indeed, all moral standards are relevant to considers identifying with HIV/AIDS. Nonetheless, the moves characteristic to AIDS-sway research necessitates that moral contemplations stretch out past the biomedical applications and that the morals. Financial effect research that are significant and appropriate to the circumstance. This implies that network sensitization is required prior to getting educated assent just as cautious thought to talking equivalent quantities of influenced and non-influenced family units or applying random sampling strategies to evade social naming. Additionally, any advantages got from sway concentrates as work intercessions or in-kind and monetary help ought to receive a network based methodology that ought to likewise consider evading belittling and separation because of inconsistent circulation of advantages and weights among network individuals.

\section{Conclusion}

The effect of AIDS is assorted and reliant on a wide scope of variables. Disregarding the variety of effects can prompt the definition of unseemly moderation gauges that don't emulate the assorted requirements among influenced family units. Consequently, examination ought to separate its example populace as per deliberately chose measures like sexual orientation and job of the individual sick or perished and, test size allowing, pre-demise resource level to comprehend the differential effects of AIDS. HIV/AIDS effects affects individuals' occupations and triggers a dynamic cycle of family reactions, which can't be perceived by taking a preview however requires a longitudinal methodology. It likewise calls for more joint effort between scientists in utilizing each other's testing casings to take into account subsequent examination. Thusly, review based investigations produce information that can be experimentally noticed and estimated however by its temperament don't test all the more profoundly into individuals' records of public activity. Given the affectability of the exploration subject, a coordinated examination plan that arrangements subjective and quantitative examination strategies is fundamental to the achievement of a HIV/AIDS study.
The intricate idea of HIV/AIDS calls for sway studies to disaggregate their information by various elements, which implies the example ought to be of sufficient size to permit various kinds of effect examinations. Then again, AIDS-sway considers utilize nonrandom strategies for information assortment to guarantee satisfactory portrayal of influenced families, for example, enlisting respondents through medical care offices or NGOs and CBOs that give HIV/AIDS-related administrations. Be that as it may, this raises moral worries as family units probably won't have given to the wellbeing place to impart their status to other people or probably won't have the foggiest idea about their status. Helps associates with an intricate arrangement of ecological, socioconservative and political components. This attribution issue can be somewhat tackled by utilizing a changed verbal dissection technique and intermediary markers to build unwavering quality in distinguishing influenced families, also by including non-affected family units as a benchmark group. Information on HIV related issues assembled from for example segment wellbeing reviews could be connected to information got from public agrarian censuses and public pay and uses overviews. In this way, rather than restricting revenue in HIV/AIDS measurements to area explicit investigations, there is a dire need to coordinate HIV/AIDS issues in public factual frameworks.

\section{References}

1. Mirken B. The ethical and social issues. Bulletin of experimental treatment for AIDS. San Francisco AIDS Foundation; Summer/autumn 2002. AIDS Vaccine; pp. 6-8

2. Bose Pradip Kumar: Research Methodology - A Trend Report, Research in Sociology and Social Anthropology - Monograph 4.

3. rady $C$. Human immunodeficiency disease; ethical considerations for clinicians. In: DeVita VT Jr, Hellman $\mathrm{S}$, Rosenberg SA, editors. AIDS. Etiology, Diagnosis, Treatment and Prevention. 4thed. Lippincort-Raven; 1977. pp. 633-642.

4. Wiegers, E.S. (2003). 'Asset Dynamics and HIV/AIDS: Household Livelihood Research Framework'. Improving Rural Livelihoods of HIV/AIDS-affected Households in Northern Province project, Zambia. Rome: FAO.

5. Wilk, R.R. and R.McC. Netting (1984). 'Households: Changing Forms and Functions'. In: R.McC. Netting, R.R. Wilk and E.J. Arnould (eds) Households: Comparative and Historical Studies of the Domestic Group, pp. 1-28. Berkeley: University of California Press.

6. Urassa, M., J.T. Boera and R. Isingo (2001). 'The impact of HIV/AIDS on mortality and household mobility in rural Tanzania', AIDS 15: 2017-2023.

7. Maxwell, David (2006). African gifts of the spirit: Pentecostalism and the rise of a Zimbabwean transnational movement. Ohio: Ohio University Press.

8. McCall, George J. (2006). The fieldwork tradition. In 
Dick Hobbs \& Richard Wright (Eds.), The Sage handbook of fieldwork (pp.3-21). London: Sage.

9. Merriam Sharan B.; Johnson-Bailey, Juanita; Lee, Ming-Yeh; Kee, Youngwha; Ntseane, Gabo \& Muhamad, Mazanah (2001). Power and positionality: Negotiating insider/outsider status within and across cultures. International Journal of Lifelong Education, 20(5), 405-416.

10. Monahan, Torin \& Fisher, Jill A. (2010). Benefits of "observer effects": Lessons from the field. Qualitative Research, 10(3), 357-376. 